\title{
Fragmentation and abrasion in granular matter systems
}

\author{
Thomas Weinhart ${ }^{1}$. Jeremy Lechman ${ }^{2}$. Thorsten Pöschel ${ }^{3}$
}

Published online: 6 November 2021

(c) The Author(s) 2021

Fragmentation and abrasion are fundamental particle-level processes that affect many macroscopic properties of granular media, from the particle size distribution to the stressstrain relationship to the wear of process equipment. Studying those processes is essential in many fields, including the geotechnical engineering, mining, pharmaceutical, and chemical industries.

Fragmentation and abrasion in granular systems can be described on at least two complementary scales. On the intra-particle scale, one needs to understand the fracture behavior of a single particle. Such fractures are affected by the material properties and the internal structure of the particle. Pore structure, pre-cracks, material plasticity, and the particles' geometric shape affect the particles' fracture behavior. Modeling the intra-particle scale allows characterizing the fragmentation behavior of granules, resulting in fracture models for multi-particle simulations. As particle properties vary within a granular bulk, usually those fracture models rely on a statistical approach; thus, they describe the average fracture behavior of a particle. Fracture models derived in this way can then be used to understand the macroscopic behavior of the bulk material.

Various numerical approaches have been used to simulate particle fragmentation and abrasion, such as peridynamics, the discrete element method (DEM), and contact dynamics. As with all numerical models, experimental calibration and validation are essential to the development of practical and predictive simulation methods.

\footnotetext{
Thorsten Pöschel

thorsten.poeschel@fau.de

Thomas Weinhart

t.weinhart@utwente.nl

Jeremy Lechman

jblechm@sandia.gov

1 Thermal and Fluid Engineering (ET), University of Twente, Enschede, The Netherlands

2 Sandia National Laboratories, Albuquerque, NM 87185, USA

3 Institute for Multiscale Simulation, Friedrich-Alexander University, Erlangen, Germany
}

We have asked several experts in modeling fragmentation and abrasion in granular systems to contribute to the present issues. The topics of these contributions are vast, ranging from intra-particle-scale simulations to calibration and validation to the development of numerical methods to predict bulk-scale behaviour. In the first paper, the authors present new peridynamics model for modeling molecular crystal particles. The second paper discusses the effects of pre-cracks in the internal structure of the particles on the fracture behavior using peridynamics. In the third paper, the authors apply a constrained DEM model to predict the effect of pore size distribution on fracture behaviour. The fourth article shows that a calibrated DEM bond model can be used to predict stress-strain relationships and dust formation in a bulk sample. The authors of the fifth paper show how a DEM model can be used to predict the abrasive wear of an impeller in a tumbler. The sixth article analyzes experimental data of gravel rock particles that can be used to calibrate fracture models. Finally, in the last paper, the authors use a coupled peridynamics-contact dynamics model to predict the particle size and shape distribution in a granular sample.

The articles compiled in this topical issue highlight many open challenges in this field: The breakage and abrasion behavior of a granular material depend strongly on the individual particles' characteristics and structure. A careful calibration and model selection are essential to obtain reliable predictions from numerical simulations. We hope that the scientific articles presented in this issue will motivate scientists working in this field.

Funding Open Access funding enabled and organized by Projekt DEAL.

Open Access This article is licensed under a Creative Commons Attribution 4.0 International License, which permits use, sharing, adaptation, distribution and reproduction in any medium or format, as long as you give appropriate credit to the original author(s) and the source, provide a link to the Creative Commons licence, and indicate if changes were made. The images or other third party material in this article are included in the article's Creative Commons licence, unless indicated otherwise in a credit line to the material. If material is not included in the article's Creative Commons licence and your intended use is not permitted by statutory regulation or exceeds the 
permitted use, you will need to obtain permission directly from the copyright holder. To view a copy of this licence, visit http://creativecomm ons.org/licenses/by/4.0/.
Publisher's Note Springer Nature remains neutral with regard to jurisdictional claims in published maps and institutional affiliations. 